\title{
Utilidad de los niveles séricos de bilirrubina en el diagnóstico de apendicitis aguda
}

\author{
Usefulness of bilirubin serum levels in the diagnosis of acute appendicitis
}

Ernesto Javier Palma-Ramírez, ${ }^{* * * *}$ Mario Enrique Rendón-Macías*,***

Palabras clave: Bilirrubinas, apendicitis, diagnóstico.

Key words: Bilirubins, appendicitis, diagnosis.

* Facultad Mexicana de Medicina, División de Postgrado, Universidad La Salle.

** Departamento de Cirugía General, Nuevo Sanatorio Durango.

*** Universidad La Salle. Unidad de Investigación en Epidemiología Clínica, Hospital de Pediatría, Centro Médico Nacional Siglo XXI, Instituto Mexicano del Seguro Social.

Recibido: 01/06/2015 Aceptado: 27/10/2017

\section{RESUMEN}

Introducción: Aun con la disponibilidad de escalas (Alvarado) para mejorar la certeza diagnóstica en la apendicitis aguda, persiste la realización de cirugías incidentales, diagnóstico y tratamiento retardado. Objetivo: Determinar la utilidad de la medición de las bilirrubinas séricas para mejorar el diagnóstico de apendicitis aguda. Material y método: Pacientes mayores de 18 años, con diagnóstico de sospecha de apendicitis aguda (Alvarado de cinco a ocho), que cumplieran los criterios de inclusión. Se les determinaron los niveles de bilirrubina sérica total (BT), indirecta (BI) y directa (BD) previos a la cirugía. El diagnóstico definitivo fue histopatológico, o evolución clínica en los no quirúrgicos. Se realizaron curvas ROC (curva del receptor operante) para evaluar el desempeño y el mejor punto de corte en cada prueba y determinar la sensibilidad y especificidad. Resultados: Fueron incluidos 86 pacientes, $52(60 \%)$ con diagnóstico confirmado de apendicitis aguda. Sesenta y cinco $(75 \%)$ requirieron de manejo quirúrgico (56 laparotomía y nueve laparoscopia); en $13(38 \%)$, la cirugía fue incidental. En el mejor nivel de corte, la BT ( $\geq 1 \mathrm{mg} / \mathrm{dl}$ ) tuvo una sensibilidad de $79.4 \%$ y una especificidad de $67.2 \%$; para la $\mathrm{BI},(\geq 0.6 \mathrm{mg} / \mathrm{dl})$, una sensibilidad de $65.5 \%$ y especificidad de $73.6 \%$; y para BD $(\geq 0.2 \mathrm{mg} / \mathrm{dl}$ ), una sensibilidad de $70.6 \%$ y especificidad de $76.9 \%$. Niveles de confirmación diagnóstica: $\mathrm{BT} \geq 1.8$ $\mathrm{mg} / \mathrm{dl}, \mathrm{BI} \geq 1.5 \mathrm{mg} / \mathrm{dl}$ y $\mathrm{BD} \geq 0.5 \mathrm{mg} / \mathrm{dl}$. Conclusiones: Los niveles séricos de bilirrubinas pueden ser de utilidad diagnóstica, sobre todo en cifras elevadas.
Introduction: Even with the availability of scales (Alvarado) to improve diagnostic accuracy in acute appendicitis, incidental performing surgeries and delayed diagnosis and treatment persist. Objective: To determine the usefulness of measuring serum bilirubin to improve the diagnosis of acute appendicitis. Material and method: Patients older than 18 years, diagnosed with suspected acute appendicitis (Alvarado 5-8) satisfying the inclusion criteria. Their levels of total serum bilirubin (TB), indirect $(I B)$ and direct $(D B)$ bilirubins were determined before surgery. The definitive diagnosis was histopathological, or depended on the clinical course in nonsurgical patients. ROC (receptor operating curve) curves were used to evaluate the performance and the best cut point in each test, and to determine the sensitivity and specificity. Results: Eighty-six patients were included, 52 (60\%) with a confirmed diagnosis of acute appendicitis. Sixty-five (75\%) required surgical management (56 laparoscopic surgery, nine laparotomy); in $13(38 \%)$, surgery was incidental. The best cut level for $T B(\geq 1 \mathrm{mg} / \mathrm{dl})$ had a sensitivity of $79.4 \%$ and a specificity of $67.2 \%$; for $I B(\geq 0.6 \mathrm{mg} / \mathrm{dl})$, a sensitivity of $65.5 \%$ and specificity of $73.6 \%$; and $D B(\geq 0.2 \mathrm{mg} / \mathrm{dl})$, a sensitivity of $70.6 \%$ and specificity of $76.9 \%$. Levels of diagnostic confirmation: $T B \geq 1.8 \mathrm{mg} / \mathrm{dL}, I B \geq 1.5 \mathrm{mg} / \mathrm{dl}$ and $D B \geq 0.5 \mathrm{mg} / \mathrm{dl}$. Conclusion: Serum bilirubin levels can be diagnostically useful, especially in high numbers.

\section{INTRODUCCIÓN}

$\mathrm{L}$ a apendicitis es la causa quirúrgica abdominal más común, estimada en 10 casos por cada 10 mil pacientes/año. ${ }^{1,2}$ Tiene una mortalidad de $0.3 \%$, la cual se incrementa a $6.5 \%$ en casos de perforación; se ha relacionado con riesgo de infertilidad en $4.8 \%$ para mujeres que nunca han estado embarazadas y $3.2 \%$ en mujeres con antecedente de embarazo, en casos de ruptura apendicular. ${ }^{3,4}$ Se estima que a un $12 \%$ de los varones y $25 \%$ de las mujeres se les realizará una apendicectomía por apendicitis aguda. ${ }^{1,5}$ Esta última ocurre sobre todo en varones en la segunda a cuarta décadas de la vida; en promedio, a los 31.3 años. ${ }^{5}$

El resultado exitoso de una apendicectomía por apendicitis está en relación con su detección antes de gangrenarse o perforarse. ${ }^{6}$ Aunque la detección depende de la pericia del cirujano, se han desarrollado escalas que permiten determinar el grado de certeza 
diagnóstica, tal es el ejemplo de la escala de Alvarado (sensibilidad y especificidad del 97\%), la cual considera ocho criterios y se califica en una escala de 10 puntos. Un puntaje de uno a tres sugiere descartar la posibilidad de apendicitis, un puntaje de cuatro a ocho aconseja vigilancia y realización de estudios complementarios como ultrasonido, tomografía axial computada abdominal o resonancia magnética. Finalmente, un puntaje de nueve o 10 recomienda la realización del procedimiento quirúrgico. Su aplicación ha permitido reducir las apendicectomías incidentales en menos del $5 \%$ de los casos. ${ }^{7}$

La duda de la decisión quirúrgica persiste en pacientes con puntajes de cuatro a ocho, en quienes se han sugerido opciones como los estudios de imagen; sin embargo, estos aún mantienen una sensibilidad baja; tal es el caso del ultrasonido, con un estimado de 55-96\% (dependiendo del observador). La tomografía axial computada (TAC) y la resonancia magnética nuclear (RMN), aunque con una mejor sensibilidad (entre 90 a 98\%), incrementan los costos y tiempos de atención, ${ }^{8-11}$ y aun se asocian hasta en un $3 \%$ a diagnósticos erróneos de apendicitis o retardo, con cirugías de urgencia por ruptura apendicular. ${ }^{11}$

Es de mencionar que existen factores que incrementan el riesgo de diagnósticos erróneos, tales como la edad de los pacientes (mayor riesgo en los extremos de la vida), ser mujer (22 contra $9.3 \%$ para hombres) y presencia de comorbilidades. ${ }^{5,12,13}$

Por lo anterior, se ha propuesto la utilización de otros marcadores bioquímicos tales como la procalcitonina sérica, el dímero-D y la proteína$\mathrm{C}$ reactiva. ${ }^{14}$ Todos ellos tienen un valor diagnóstico aceptable, aunque no concluyente, y la limitante de no estar disponibles en muchos centros de atención.

Un marcador biológico potencial ha sido la bilirrubina sérica, la cual se ha encontrado en pacientes con apendicitis asociada a la liberación de toxinas de E. coli y Bacteroides fragilis (bacterias encontradas en las paredes del apéndice), las cuales han mostrado reducir el flujo biliar en modelos de roedores in vivo. Además, la ictericia es frecuente en la sepsis, sobre todo asociada a la existencia de bacterias Gram negativas y a hemólisis secundaria, lo que causa una carga de bilirrubina incrementada, mecanismo asociado con varias bacterias, incluyendo E. coli. ${ }^{15-17}$ Otros mecanismos son la captación hepática reducida y la excreción canalicular de bilirrubina causada por endotoxemia. ${ }^{18,19}$ Las endotoxinas bacterianas causan inhibición mediada por citocinas de los mecanismos de transporte de sales biliares y llevan a colestasis. ${ }^{18,20,21}$

En el presente trabajo definimos como 'caso probable' a pacientes con Alvarado de entre cinco y ocho puntos, a cuyo ingreso se les solicitaron bilirrubinas séricas; se pretende agregar el valor diagnóstico de las bilirrubinas a la escala de Alvarado para normar una conducta terapéutica oportuna.

\section{MATERIAL Y MÉTODO}

Se realizó un estudio transversal para identificar casos y controles. La investigación se llevó a cabo de enero de 2012 a abril de 2014 en pacientes hospitalizados en el Nuevo Sanatorio Durango. De un total de 176 pacientes con sospecha de apendicitis aguda, se excluyeron a 90 por presentar alcoholismo, hepatitis viral, embarazo, enfermedades hemolíticas, hepáticas o de las vías biliares; síndrome de Gilbert o de Dubin Johnson; asimismo, pacientes con valoración de la escala de Alvarado menor a cinco o mayor a ocho, y aquellos sin determinación sérica de bilirrubinas.

El diagnóstico de sospecha de apendicitis y la valoración de la escala de Alvarado fueron realizados por la anamnesis y exploración física del cirujano. En los casos de cirugía, esta fue realizada de manera abierta o por vía laparoscópica; en todos los casos se hizo un estudio histopatológico para confirmar el diagnóstico clínico.

Los 86 pacientes incluidos en el estudio pudieron tener resolución clínica no quirúrgica (control), apendicectomía incidental (control), apendicitis fase I (hiperémica, edematosa), apendicitis fase II (abscedada), apendicitis fase III (necrosada) o apendicitis fase IV (perforada con peritonitis). Además de la valoración de la escala de Alvarado (migración del dolor, anorexia, náusea o vómito, hipersensibilidad, fiebre, leucocitosis y neutrofilia), a estos pacientes se les determinaron las bilirrubinas séricas 
al ingreso al Servicio de Urgencias. De todos se obtuvo su sexo, edad, días de evolución y medicación (en particular, antibióticos) previos a su ingreso hospitalario. La recolección de datos fue registrada en una base de Microsoft Excel para Windows.

El análisis estadístico fue realizado utilizando el programa SPSS 20.0 para Windows. La hipótesis nula fue asumir que no habría diferencia en los niveles de bilirrubina entre los pacientes con y sin apendicitis aguda. Los valores de media, desviación estándar, rangos y valores de $\mathrm{P}$ fueron calculados para sexo, edad, escala de Alvarado, días de evolución previa, diagnóstico histopatológico, tipo de cirugía, con la prueba exacta de Fisher a dos colas, t de Student a dos colas, chi cuadrada lineal por lineal y $U$ de Mann Whitney. Se realizaron curvas ROC para evaluar el desempeño y el mejor punto de corte en cada prueba y determinar la sensibilidad y especificidad de la determinación de bilirrubinas. Los cocientes de riesgo fueron realizados para bilirrubina total, indirecta y directa a través de una operación de regresión logística binaria después de un análisis multivariable de datos. Un valor de $\mathrm{p}$ $<0.05$ fue usado para determinar significancia estadística.

\section{Cuadro I. Descripción de las características de los pacientes según su diagnóstico final.}

\begin{tabular}{|c|c|c|c|c|c|}
\hline \multirow{2}{*}{$\begin{array}{l}\text { Datos } \\
\text { Sexo }\end{array}$} & \multicolumn{2}{|c|}{$\begin{array}{l}\text { Pacientes con diagnóstico } \\
\text { de apendicitis aguda } \\
n=52(\%)\end{array}$} & \multicolumn{2}{|c|}{$\begin{array}{l}\text { Pacientes con otras } \\
\text { enfermedades } \\
n=34(\%)\end{array}$} & \multirow[t]{2}{*}{ Valor de $p$} \\
\hline & & & & & \\
\hline Masculino & 29 & $(55.8)$ & 15 & $(44.1)$ & $0.38 *$ \\
\hline Femenino & 23 & $(44.2)$ & 19 & $(55.9)$ & \\
\hline Edad, media $\pm 1 \mathrm{DE}$ & 42.8 & \pm 2 & 42 & \pm 3 & $0.81 * *$ \\
\hline \multicolumn{6}{|c|}{ Puntaje de la escala de Alvarado } \\
\hline 5 & 3 & $(5.8)$ & 18 & $(52.9)$ & $<0.001^{+}$ \\
\hline 6 & 11 & $(21.2)$ & 8 & $(23.5)$ & \\
\hline 7 & 20 & $(38.5)$ & 3 & $(8.8)$ & \\
\hline 8 & 18 & $(34.6)$ & 5 & $(14.7)$ & \\
\hline \multicolumn{6}{|c|}{ Días de evolución previos } \\
\hline Mediana (límites) & 1 & $(0-13)$ & 1 & $(0-32)$ & $0.48^{++}$ \\
\hline \multicolumn{6}{|l|}{ Medicación previa } \\
\hline Sí & 35 & $(67.3)$ & 20 & $(58.8)$ & $0.49 *$ \\
\hline Antibiótico & 6 & $(11.5)$ & 7 & $(20.6)$ & $0.35^{*}$ \\
\hline \multicolumn{6}{|c|}{ Diagnóstico histopatológico } \\
\hline No cirugía & & & 21 & $(61.8)$ & \\
\hline Incidental & & & 13 & $(38.2)$ & \\
\hline Fase I & 3 & $(5.8)$ & & & \\
\hline Fase II & 14 & $(26.9)$ & & & \\
\hline Fase III & 16 & $(30.8)$ & & & \\
\hline Fase IV & 19 & $(36.5)$ & & & \\
\hline \multicolumn{6}{|l|}{ Tipo de cirugía } \\
\hline Laparotomía & 47 & $(90.4)$ & 9 & $(69.3)$ & $0.07 *$ \\
\hline Laparoscopia & 5 & $(9.6)$ & 4 & $(30.7)$ & \\
\hline No se realizó & - & - & 21 & - & \\
\hline
\end{tabular}

Nota $=*$ Prueba exacta de Fisher de dos colas. ** Prueba t de Student dos colas, + Prueba chi cuadrada lineal por lineal, ++ Prueba U de Mann Whitney. 


\section{RESULTADOS}

De los 86 pacientes incluidos en el estudio, $42(49 \%)$ fueron mujeres y 44 (51\%) hombres. Cincuenta y dos tuvieron confirmación de apendicitis aguda (60\%), 29 (56\%) hombres y $23(44 \%)$ mujeres. Los restantes $34(40 \%)$ presentaron otras enfermedades, 15 (44\%) hombres y 19 (56\%) mujeres. Sesenta y cinco (75\%) requirieron de manejo quirúrgico (56 laparotomía y nueve laparoscopia); en 13 (38\%) la cirugía fue incidental. No hubo diferencia estadísticamente significativa entre sexos ( $p$ $=0.38$ ). La edad media entre pacientes con apendicitis fue de 42.8 años ( $D E \pm 2$,) y la de aquellos con otras enfermedades fue de 42 ( $\mathrm{DE} \pm 3$ ). Los pacientes con apendicitis tuvieron puntajes más altos de Alvarado, $\mathrm{p}$ $<0.001$ (Cuadro I). No hubo diferencias en relación con la medicación previa y el uso de antibióticos, ni con el tiempo de evolución. Aunque en el grupo control hubo mayor proporción de cirugías laparoscópicas, esta diferencia no alcanzó la significancia estadística, $\mathrm{p}=0.07$ (Cuadro I).

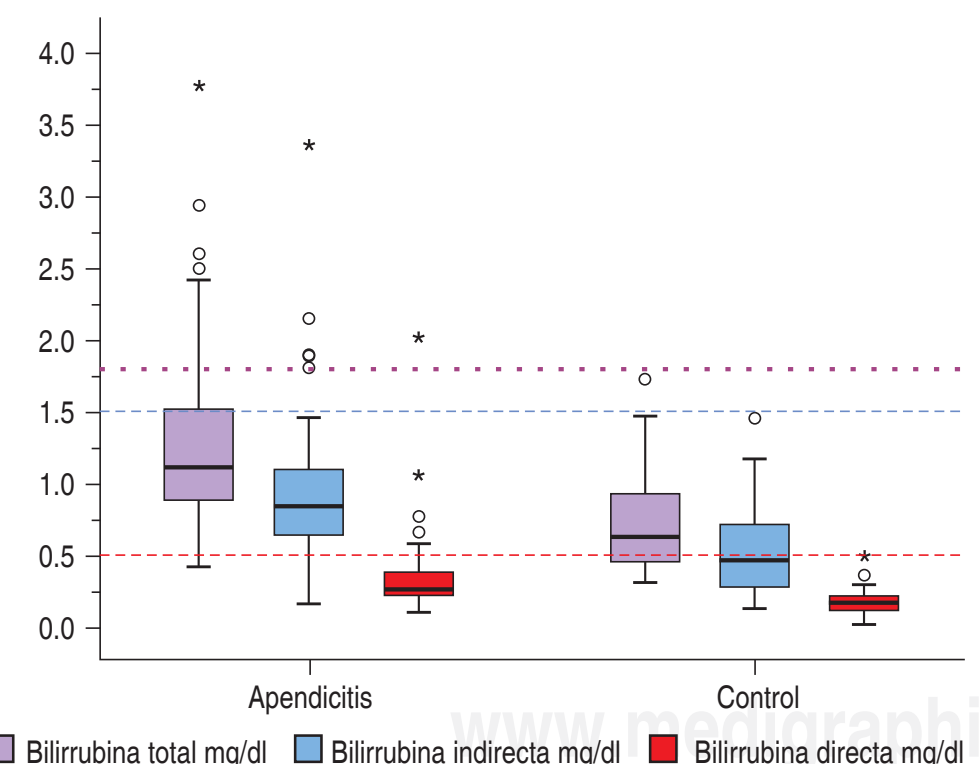

Las líneas horizontales de las cajas muestran la mediana; la caja equivale al cuartil 1 y 3 ; los bigotes, a los centiles 10 y 90; círculos y estrellas, a valores extremos y muy extremos. Las líneas punteadas muestran los límites de confirmación diagnóstica para bilirrubina total (morada), indirecta (azul) y directa (roja).

Figura 1. Distribución de los valores de bilirrubina total, indirecta y directa según la presencia o no de apendicitis aguda.
Como se muestra en la figura 1 , los niveles de bilirrubina sérica fueron más altos en los pacientes con apendicitis; la mediana en ellos fue de $1.2 \mathrm{mg} / \mathrm{dl}$ contra $0.7 \mathrm{mg} / \mathrm{dl}$ en los controles, $U$ de Mann-Whitney $p=0.03$. Lo mismo sucedió con los niveles de bilirrubina directa (mediana $0.7 \mathrm{mg} / \mathrm{dl}$ contra $0.5 \mathrm{mg} / \mathrm{dl}$, $p=0.01)$.

El mejor desempeño fue para la BT sérica [área bajo la curva, $\mathrm{ABC}=0.81\left(\mathrm{IC}_{95 \%} ; 0.71\right.$ 0.90)], seguida de la $\mathrm{BI}\left[\mathrm{ABC}=0.77\left(\mathrm{IC}_{95 \%} ;\right.\right.$ $0.67-0.88)]$ y la $\mathrm{BD}\left[\mathrm{ABC}=0.74\left(\mathrm{IC}_{95 \%} ; 0.63-\right.\right.$ 0.85)] (Figura 2). En el mejor nivel de corte, la BT $(1 \mathrm{mg} / \mathrm{dl}$ ) tuvo una sensibilidad de $79.4 \%$ $\left(\mathrm{IC}_{95 \%} ; 64-94\right)$ y una especificidad de $67.3 \%$ $\left(\mathrm{IC}_{95 \%} ; 53-81\right)$; para la BI $(0.6 \mathrm{mg} / \mathrm{dl})$, una sensibilidad de $65.5 \%\left(\mathrm{IC}_{95 \%} ; 46-84\right)$ y especificidad de $73.6 \%\left(\mathrm{IC}_{95 \%} ; 61-86\right)$; y para la $\mathrm{BD}(0.2 \mathrm{mg} /$ $\mathrm{dl})$, una sensibilidad de $70.6 \%\left(\mathrm{IC}_{95 \%} ; 53-87\right)$ y especificidad de $76.9 \%\left(\mathrm{IC}_{95 \%} ; 64-89\right)$ (Cuadro II). Niveles de confirmación: BT $\geq 1.8 \mathrm{mg} / \mathrm{dl}$, $\mathrm{BI} \geq 1.5 \mathrm{mg} / \mathrm{dl}$ y $\mathrm{BD} \geq 0.5 \mathrm{mg} / \mathrm{dl}$.

\section{DISCUSIÓN}

Nuestros datos muestran que la determinación de los niveles de bilirrubina sérica fueron más altos en los pacientes con apendicitis aguda y

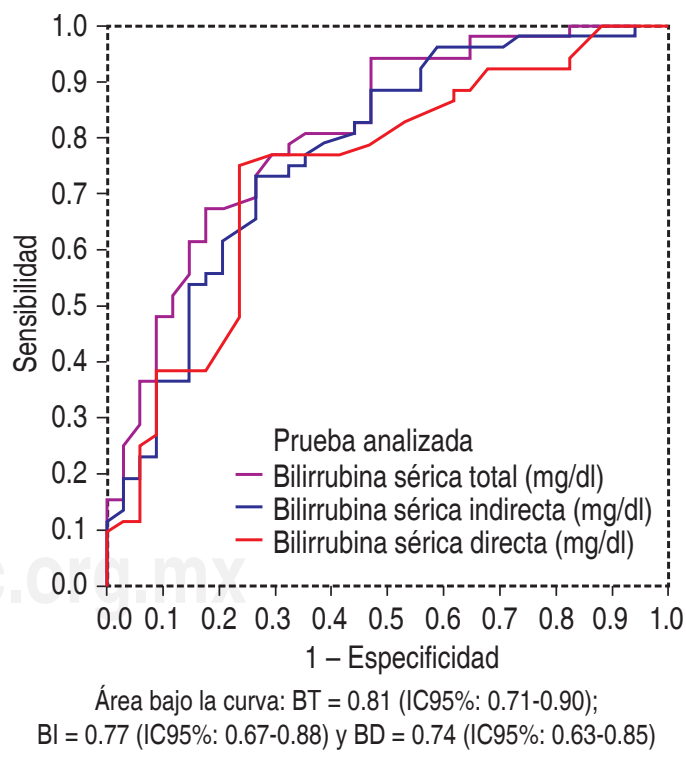

Figura 2. Curvas ROC del desempeño de la medición de la bilirrubina total, indirecta y directa para el diagnóstico de apendicitis aguda. 
Cuadro II. Análisis de validez de la determinación de los valores de bilirrubinemia para el diagnóstico de apendicitis aguda.

\begin{tabular}{|c|c|c|c|}
\hline Índices & $\begin{array}{l}\text { Bilirrubina total } \\
\text { Nivel corte } \\
1 \mathrm{mg} / \mathrm{dl}\end{array}$ & $\begin{array}{c}\text { Bilirrubina indirecta } \\
\text { Nivel de corte } \\
0.6 \mathrm{mg} / \mathrm{dl}\end{array}$ & $\begin{array}{l}\text { Bilirrubina directa } \\
\text { Nivel de corte } \\
0.2 \mathrm{mg} / \mathrm{dl}\end{array}$ \\
\hline Verdaderos positivos & 27 & 19 & 24 \\
\hline Verdaderos negativos & 35 & 42 & 40 \\
\hline Falsos positivos & 17 & 10 & 12 \\
\hline Falsos negativos & 7 & 15 & 10 \\
\hline Sensibilidad \% & 79.4 (64 a 94) & $65.5(46$ a 84$)$ & 70.6 (53 a 87) \\
\hline Especificidad \% & $67.3(53$ a 81$)$ & $73.6(61$ a 86$)$ & $76.9(64$ a 89$)$ \\
\hline Valor predictivo positivo $\%$ & $61(45$ a 76$)$ & $56(38$ a 74$)$ & $67(50$ a 83$)$ \\
\hline Valor predictivo negativo \% & 83 (70 a 96) & $81(69$ a 92$)$ & $80(68$ a 92$)$ \\
\hline Razón de probabilidad positiva & $2.4(1.6$ a 3.7$)$ & $2.5(1.5$ a 4$)$ & $3(1.8$ a 5.6$)$ \\
\hline Razón de probabilidad negativa & $0.3(0.15$ a 0.6$)$ & $0.5(0.3$ a 0.8$)$ & $0.4(0.2$ a 0.7$)$ \\
\hline Punto de confirmación diagnóstica & $\geq 1.8 \mathrm{mg} / \mathrm{dl}$ & $\geq 1.5 \mathrm{mg} / \mathrm{dl}$ & $\geq 0.5 \mathrm{mg} / \mathrm{dl}$ \\
\hline OR diagnóstica & $7.9(2.9$ a 21$)$ & $4.2(1.6$ a 10.6$)$ & $8(3$ a 21$)$ \\
\hline
\end{tabular}

confirmatorios a un nivel de BT $\geq 1.8 \mathrm{mg} / \mathrm{dl}$. Estudios previos ${ }^{13,15}$ ya habían observado la elevación de este metabolito en la sangre de estos pacientes, pero nuestro trabajo permitió establecer las cifras y su validez en el diagnóstico.

$\mathrm{Si}$ bien otros marcadores como la procalcitonina, dímero-D y proteína- $C$ reactiva han sido usados en el diagnóstico de apendicitis aguda, con buenos indicadores de validez diagnóstica, ${ }^{3,14}$ tienen como principal limitante su disponibilidad. Consideramos que la determinación de bilirrubinas séricas se puede realizar en cualquier hospital de segundo nivel o general, incluso en algunas clínicas de primer contacto. Asimismo, el costo de esta determinación al momento actual es muy inferior a los anteriores.

Un aspecto a recalcar fue la observación de que, aunque nuestros pacientes con apendicitis aguda tuvieron, en general, puntajes más altos en la escala de Alvarado, los niveles de bilirrubina no se correlacionaron (Rho Spearman $=$ 0.12); por ello, pacientes con poca sintomatología pueden ser vigilados o explorados a mayor detalle si se les encuentran niveles altos de bilirrubina.

Por otro lado, aunque el desempeño de los niveles séricos de bilirrubina sérica tuvo un área bajo la curva por arriba de 0.70 , consideramos que no pueden ser tomados como dato único para el establecimiento de un diagnóstico de apendicitis. El criterio encontrado para esta seguridad fue con un nivel superior a $1.8 \mathrm{mg} / \mathrm{dl}$ para bilirrubina total, $1.5 \mathrm{mg} / \mathrm{dl}$ para bilirrubina indirecta y $0.5 \mathrm{mg} / \mathrm{dl}$ para bilirrubina directa.

Nuestro estudio tuvo la fortaleza de disponer de un amplio grupo de pacientes en cuanto a su sintomatología y la gravedad de sus apendicitis. Asimismo, las valoraciones fueron independientes de la decisión quirúrgica y se cuidó eliminar a pacientes con otras enfermedades asociadas con elevación de las bilirrubinas. Por otro lado, nuestros datos no permitieron establecer si los niveles de bilirrubina podrían predecir el nivel de gravedad de la apendicitis. Aún es necesario evaluar su utilidad en pacientes en donde el diagnóstico es más difícil, como son los niños y los ancianos.

\section{CONCLUSIONES}

Los niveles séricos de bilirrubinas son de utilidad diagnóstica de apendicitis aguda, sobre todo si estos se encuentran $\geq 1.8 \mathrm{mg} / \mathrm{dl}$ para 
la bilirrubina total, $\geq 1.5 \mathrm{mg} / \mathrm{dl}$ para bilirrubina indirecta $y \geq 0.5 \mathrm{mg} / \mathrm{dl}$ para bilirrubina directa.

\section{REFERENCIAS}

1. Korner H, Söndenaa K, Söreide JA, Andersen E, Nysted A, Lende TH, et al. Incidence of acute non perforated and perforated appendicitis: age-specific and sex specific analysis. World J Surg. 1997; 21: 313-317.

2. Flum DR, Morris A, Koepsell T, Dellinger EP. Has misdiagnosis of appendicitis decreased over time? A population-based analysis. JAMA. 2001; 286: 17481753.

3. Schwarz A, Bolke E, Peiper M, Schulte am Esch J, Steinbach G, van Griensven M, et al. Inflammatory peritoneal reaction after perforated appendicitis: continuous peritoneal lavage versus non lavage. Eur J Med Res. 2007; 12: 200-205.

4. Mueller BA, Daling JR, Moore DE, Weiss NS, Spadoni LR, Stadel BV, et al. Appendectomy and the risk of tubal infertility. N Engl J Med. 1986; 315: 1506-1508.

5. Addis DG, Shaffer N, Fowler BS, Tauxe RV. The epidemiology of appendicitis and appendicectomy in the United States. Am J Epidemiol. 1990; 132: 910-925.

6. Wesson DE, Singer JI, Wiley FJ. Acute appendicitis in children: clinical manifestations and diagnosis. UpToDate. (Consultado el 4 de diciembre de 2014.

7. Escribá A, Gamell AM, Fernández Y, Quintillá JM, Cubells CL. Prospective validation of two systems of classification for the diagnosis of acute appendicitis. Pediatr Emerg Care. 2011; 27: 165-169.

8. Kaiser S, Frenckner B, Jorulf HK. Suspected appendicitis in children: US an $\mathrm{CT}$ - A prospective randomized study. Radiology. 2002; 223: 633-638.

9. Douglas CD, Macpherson NE, Davison PM, Gani JS. Randomised controlled trial of ultrasonography in diagnosis of acute appendicitis, incorporating the Alvarado score. BMJ. 2000; 321: 919-922.

10. Funaki B, Grosskreutz SR, Funaki CN. Using unenhanced helical $\mathrm{CT}$ with enteric contrast material for suspected appendicitis in patients treated at a community hospital. AJR Am J Roentgenol. 1998; 171: 997-1001.

11. Stroman DL, Bayouth CV, Kuhn JA, Westmoreland $\mathrm{M}$, Jones RC, Fisher $\mathrm{TL}$, et al. The role of computed tomography in the diagnosis of acute appendicitis. Am J Surg. 1999; 178: 485-489.

12. Flum DR, Koepsell T. The clinical and economic correlates of misdiagnosed appendicitis: Nationwide analysis. Arch Surg. 2002; 137: 799-804.

13. Noh H, Chang SJ, Han A. The diagnostic values of preoperative laboratory markers in children with complicated appendicitis. J Korean Surg Soc. 2012; 83: 237-241.

14. Kaya B, Sana B, Eris C, Karabulut K, Bat O, Kutanis R. The diagnostic value of D-dimer, procalcitonin and CPR in acute appendicitis. Int J Med Sci. 2012; 9: 909-915.

15. Whitehead MW, Hainsworth I, Kingham JG. The causes of obvious jaundice in South West Wales: perceptions versus reality. Gut. 2001; 48: 409-413.

16. Chand N, Sanyal AJ. Sepsis-induced cholestasis. Hepatology. 2007; 45: 230-241.

17. Shander A. Anemia in the critically ill. Crit Care Clin. 2004; 20: 159-178.

18. Roelofsen $H$, van der Veere $C N$, Ottenhoff $R$, Schoemaker B, Jansen PL, Oude Elferink RP. Decreased bilirubin transport in the perfused liver of endotoxemic rats. Gastroenterology. 1994; 107: 1075-1084.

19. Bolder U, Ton-Nu HT, Schteingart CD, Frick E, Hofmann AF. Hepatocyte transport of bile acids and organic anions in endotoxemic rats: impaired uptake and secretion. Gastroenterology. 1997; 112: 214-225.

20. Green RM, Beier D, Gollan JL. Regulation of hepatocyte bile salt transporters by endotoxin and inflammatory cytokines in rodents. Gastroenterology. 1996; 111: 193-198.

21. Whiting JF, Green RM, Rosenbluth AB, Gollan JL. Tumor necrosis factor-alpha decreases hepatocyte bile salt uptake and mediates endotoxin-induced cholestasis. Hepatology. 1995; 22: 1273-1278.

Correspondencia:

Ernesto Javier Palma-Ramírez

Javier Rojo Gómez MZ 53, LT44

San Agustín 1A, Chimalhuacán, 56344,

Estado de México, México.

Tel. 0445527292759

E-mail: orfeo2236@hotmail.com 